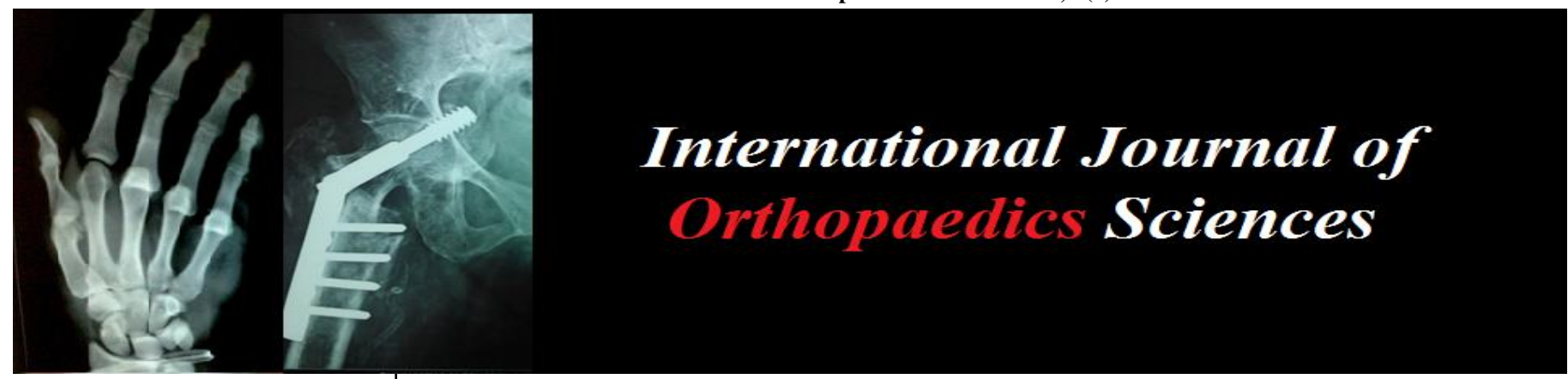

E-ISSN: 2395-1958

P-ISSN: 2706-6630

IJOS 2021; 7(2): 767-775

(C) 2021 IJOS

www.orthopaper.com

Received: 25-02-2021

Accepted: 27-03-2021

Dr. Manoj Kumar HV

Professor, Department of

Orthopedics, Bangalore Medical

College and Research Institute

(BMC\&RI), Karnataka, India

Dr. Rajendra Reddy PS

Post Graduate Student,

Department of Orthopedics,

Bangalore Medical College and

Research Institute (BMC\&RI)

Karnataka, India
Corresponding Author: Dr. Manoj Kumar HV Professor, Department of Orthopedics, Bangalore Medical College and Research Institute (BMC\&RI), Karnataka, India

\section{Infection rates following internal fixation of open and closed tibia fractures with intra medullary inter locking nail-observational cohort study}

\section{Dr. Manoj Kumar HV and Dr. Rajendra Reddy PS}

DOI: https://doi.org/10.22271/ortho.2021.v7.i2j.2704

\begin{abstract}
Background: Diaphysial fractures of tibia are most common fractures of lower extremity, this has been in raise due to increased Road Traffic Accidents (RTA). Locked intra medullary nailing currently is considered the treatment of choice. Surgical site infection following internal fixation is a fearful complication as affecting the quality of life. The primary objectives of this study were to determine the rate of infection within one year of surgery and to describe the distribution of infections by location and time of diagnosis for tibia fractures in our study.

Methods: We conducted a prospective cohort study. Patients who presented with an open or closed tibia shaft fracture treated with Inter Locking Nail (ILN) at our hospitals were invited to participate in the study. Participants attended follow-up visits at 3, 6, and 12 months post-surgery, where they were assessed for presence of infection at surgical site.

Results: 213 participants were included in the study and 199 participants completed the 12 month follow-up. The overall rates of infection within 12 months of surgery was $11 \%$ (22 participants).The incidence of infection was higher in open fractures (17.1\%) (12 infections) as compared to closed fractures $(7.8 \%)$ (10 infections) with $\mathrm{p}<0.0001$.There were $15(68 \%)$ deep infections and 7 (32\%) superficial infections. There were $8(37 \%)$ early infections, $5(23 \%)$ delayed infections, and $9(40 \%)$ late infections. Almost all participants received prophylactic antibiotics before surgery of which $(92.9 \%)$ of open fractures and $(84.5 \%)$ of patients with closed fractures received antibiotic within 60 minutes of incision and rest received more than an hour prior to surgery. All cases were given post-operative IV antibiotics $(100 \%)$. The antibiotics were given for an average of $5.5 \pm 2$ days in open fractures and $4 \pm 1$ days in closed fractures.

Conclusions: The observed infection rates in our study were much higher compared to others similar studies. The duration of prophylactic antibiotic use was longer than standard practice. It raises concern for need to look in to best practices of peri operative patient management to maintain low Infection rates and also to be vigilant about the potential development of antibiotic resistant microbes within our settings. Postoperative antibiotics for longer durations doesn't seem to reduce the infection rates as per our study.
\end{abstract}

Keywords: open and closed tibial fractures, prophylactic antibiotics, Gustilo's classification, Tscherne's classification of closed fractures, infection, inter locking nail, ESR, CRP, culture and sensitivity

\section{Introduction}

Trauma is one of the major public health problems in India as a result of accelerated urbanisation and industrialisation ${ }^{[1]}$. Fractures of tibia shaft have a recorded incidence of 17.21 per 100,000 population and it represents $36.7 \%$ of all long bone fractures in adults ${ }^{[2]}$. Diaphysial fractures of tibia are most common fractures of lower extremity. Locked intra medullary nailing currently is considered the treatment of choice for most type I, type II, and type IIIA open and closed tibial fractures. Intra medullary nailing preserves the soft tissue sleeve around the fracture site and allows early motion of the adjacent joints. The ability to lock the nails proximally and distally provides control of length, alignment, and rotation in unstable fractures and permits stabilization of fractures ${ }^{[3]}$. Surgical site infection following internal fixation is a fearful complication as it affects the quality of life along with huge socioeconomic implications on the patient. Data on the incidence of wound infection after internal fixations in developing countries are rare and report a higher incidence of infections 
(ranging from $14 \%$ to $16 \%$ ) than in industrialized countries $(3.6 \%)^{[4]}$. Currently there is not much information available exclusively on the infection pattern following intra medullary nailing of Tibia. This research was undertaken to study the infection rates following Intra medullary fixation of Tibial fractures, and also about antibiotic usage patterns in the peri operative period at our hospitals. The results obtained can be compared with the data from previous research which can be helpful in taking necessary steps at various levels in curtailing infection rates and also on deciding antibiotic protocol at our hospitals.

The primary objectives of the current study were to determine the rates of infection within one year of surgery and to describe the distribution of infections by location (superficial, deep) and time of diagnosis (early, delayed, late) for open and closed tibia fracture patients. Secondary objectives were to: to explore the effect of fracture type, time since injury to surgery, duration of antibiotics on the infection and to study various methods employed to treat infected cases.

\section{Methods}

Study overview: We conducted a prospective, observational cohort study to investigate the rates of infections within one year for open and closed tibia fracture patients who were treated with internal fixation with ILN. After obtaining informed consent, baseline and surgical data were recorded. Approval was obtained from the Institutional Ethics Committee prior to commencing study activities.

\section{Participant details and eligibility criteria}

Patients who presented to either of the participating hospitals with a tibial shaft fracture treated with ILN were screened for study eligibility. The inclusion criteria were: 1) Men and women who are 18 years of age or older with Open or closed tibial shaft fracture 2) Ability to understand the content of the subject information/informed consent form and to be willing to participate in the clinical investigation. 3) Provided written informed consent. The exclusion criteria were: 1) Previous wound infection or osteomyelitis at the same limb (according to subject history). 2) Immunological deficiency disease. 3) Tumor related fractures.

\section{Data collection}

After providing informed consent, baseline information was documented and participants underwent a haematology analysis and radiographs (AP, lateral) before surgery. Details regarding the surgical procedure, including antibiotic prophylaxis, were documented. Post-operatively participants underwent a haematology analysis and radiographs. Participants with any symptoms of surgical site infections underwent further investigations including radiological assessment, hematological analysis (ESR, CRP, Total Leucocyte Count) and bacteriological culture and antibiogram whenever possible to determine whether infection was present. If infection was diagnosed, infection management including administered antibiotics, wound care, surgical intervention performed, were recorded. Participants attended clinic visits at 3 months, 6 months, and 12 months postsurgery, or were contacted by telephone to collect as much information as possible if unable to attend a follow-up visit. At each visit, patients were assessed for infections and fracture healing. Haematology investigations and radiographs were taken as standard of care. Antibiotic use was documented.

CDC (Centre for Disease Control) criteria was used for the confirmation of the presence of infection and classify the infection as a superficial incisional surgical site infection (SSI) or a deep $\mathrm{SSI}^{5}$. And also confirmed the timing of infection as early (onset of symptoms within 2 weeks of injury), delayed (onset of symptoms 2-10 weeks after injury) or, late (onset of symptoms more than 10 weeks after injury).

\section{Data analysis}

We summarize participant characteristics using descriptive statistics expressed as means and standard deviations for continuous variables) or counts and percentages for categorical variables. For analysis of primary outcomes, the rate of infection within one year of the internal fixation was reported as a percentage. A Fisher's exact test was used to determine if the rate of infection and infection type (superficial versus deep) differed across fracture types (open versus closed fractures). Infections were classified by timing of onset and a Chi-square test was used to compare the incidence of early versus delayed versus late onset infections across fracture types. For analysis of secondary outcomes, infection symptoms and management in open and closed fracture patients were summarized using descriptive statistics. A Chi-square test was used to compare if the infection rates differed by Gustilo classification for open fractures and by Tscerne's classification for Closed fractures. Level of significance was determined as $\mathrm{p}<0.05$. Data analysis was done using Statistical Analysis. SPSS (Statistical Package for Social Sciences) version 20. [IBM SPASS statistics (IBM corp. Armonk, NY, USA released 2011)] was used to perform the statistical analysis. Data was entered in the excel spread sheet.

\section{Results}

In our study 199 of 211 patients who met the inclusion criteria and provided informed consent were followed up.12 participants who were lost for follow up are not included in the analysis. Others completed 12 months of follow up.

\section{Demographics and fracture characteristics}

The age group of maximum number of study participants was between 21 to 40 years and the majority were male (92\%) (Table 1). The majority of participants included in this study were healthy (87.9\% had no comorbidities) and non-smokers $(69 \%)$ (table 1). The most common mechanism of injury was motor vehicle accidents (95\%) (Table 1). About $20 \%$ of participants had additional injuries or fractures other than tibial fracture. The majority of participants had closed fractures (129 participants, 64.8\%), with 70 participants $(35.2 \%)$ having open fractures (table 2 ). 
Table 1: Showing Distribution of participants as per age, gender, smoking habit and Mechanism of injury leading to fracture.

\begin{tabular}{|c|c|c|}
\hline Age group & Frequency & Percent(\%) \\
\hline $18-20$ & 20 & 10.1 \\
\hline $21-40$ & 117 & 58.8 \\
\hline $41-60$ & 48 & 24.1 \\
\hline $60-80$ & 14 & 7.0 \\
\hline Total & 199 & 100.0 \\
\hline Gender & & \\
\hline Female & 17 & 8.5 \\
\hline Male & 182 & 91.5 \\
\hline Total & 199 & 100.0 \\
\hline Smoker & & \\
\hline No & 138 & 69.3 \\
\hline Yes & 61 & 30.7 \\
\hline Total & 199 & 100.0 \\
\hline Mechanism of Injury & & 2.5 \\
\hline Fall from height & 5 & .5 \\
\hline Fall in to manhole & 1 & 1.5 \\
\hline Fall of heavy object & 3 & 95.0 \\
\hline RTA & 189 & .5 \\
\hline Self fall & 1 & 100.0 \\
\hline Total & 199 & \\
\hline
\end{tabular}

Table 2: Distribution of patients as per Open and Closed fracture classification (Gustilo and Tscherne's respectively)

\begin{tabular}{|c|c|c|}
\hline Type of injury & $\begin{array}{c}\text { No of } \\
\text { patients }\end{array}$ & $\begin{array}{c}\text { Percentage } \\
(\boldsymbol{\%})\end{array}$ \\
\hline Open & 70 & 35.2 \\
\hline Closed & 129 & 64.8 \\
\hline Total & 199 & 100.0 \\
\hline $\begin{array}{c}\text { Gustilo classification for open } \\
\text { fractures (Type) }\end{array}$ & & \\
\hline Type I & 19 & 27.1 \\
\hline Type II & 32 & 45.7 \\
\hline Type IIIA & 10 & 14.3 \\
\hline Type IIIB & 9 & 12.9 \\
\hline Total & 70 & 100.0 \\
\hline $\begin{array}{c}\text { Tscherne's classification for closed } \\
\text { fractures (Type) }\end{array}$ & & \\
\hline 0 & 9 & 7.0 \\
\hline 1 & 73 & 56.6 \\
\hline 2 & 42 & 32.6 \\
\hline 3 & 5 & 3.9 \\
\hline Total & 129 & 100.0 \\
\hline & & \\
\hline & & \\
\hline & & \\
\hline & & \\
\hline & & \\
\hline & & \\
\hline & & \\
\hline & & \\
\hline & & \\
\hline & & \\
\hline & & \\
\hline
\end{tabular}

Surgical management and peri-operative care

The mean time from injury to surgery was 36 hours (Fig 1). The time from injury to surgery was $33 \pm 38 \mathrm{~h}$ for open fractures and $39.5 \pm 40 \mathrm{~h}$ for closed fractures where as it was $24+/-35 \mathrm{~h}$ for open and $27+/-39 \mathrm{~h}$ in closed fractures from admission to surgery. Almost all participants received prophylactic antibiotics before surgery of which 65 patients (92.9\%) with open fractures and $109(84.5 \%)$ of patients with closed fractures received antibiotic within 30 to 60 minutes before incision and rest that is 5 patients $(7.1 \%)$ from open fracture group and 20 patients $(12.6 \%)$ from Closed fracture group received antibiotic more than an hour (1-2 hours) prior to surgery (Fig 2)

All patients were given IV antibiotics in postoperative period for an average of $5.5 \pm 2$ (2 to 7 days) days in open fractures and 4.1 \pm 1 ( 2 to 5) days in closed fractures (Fig 3). About 194 patients received 3rd generation Cephalosporins (97\%).

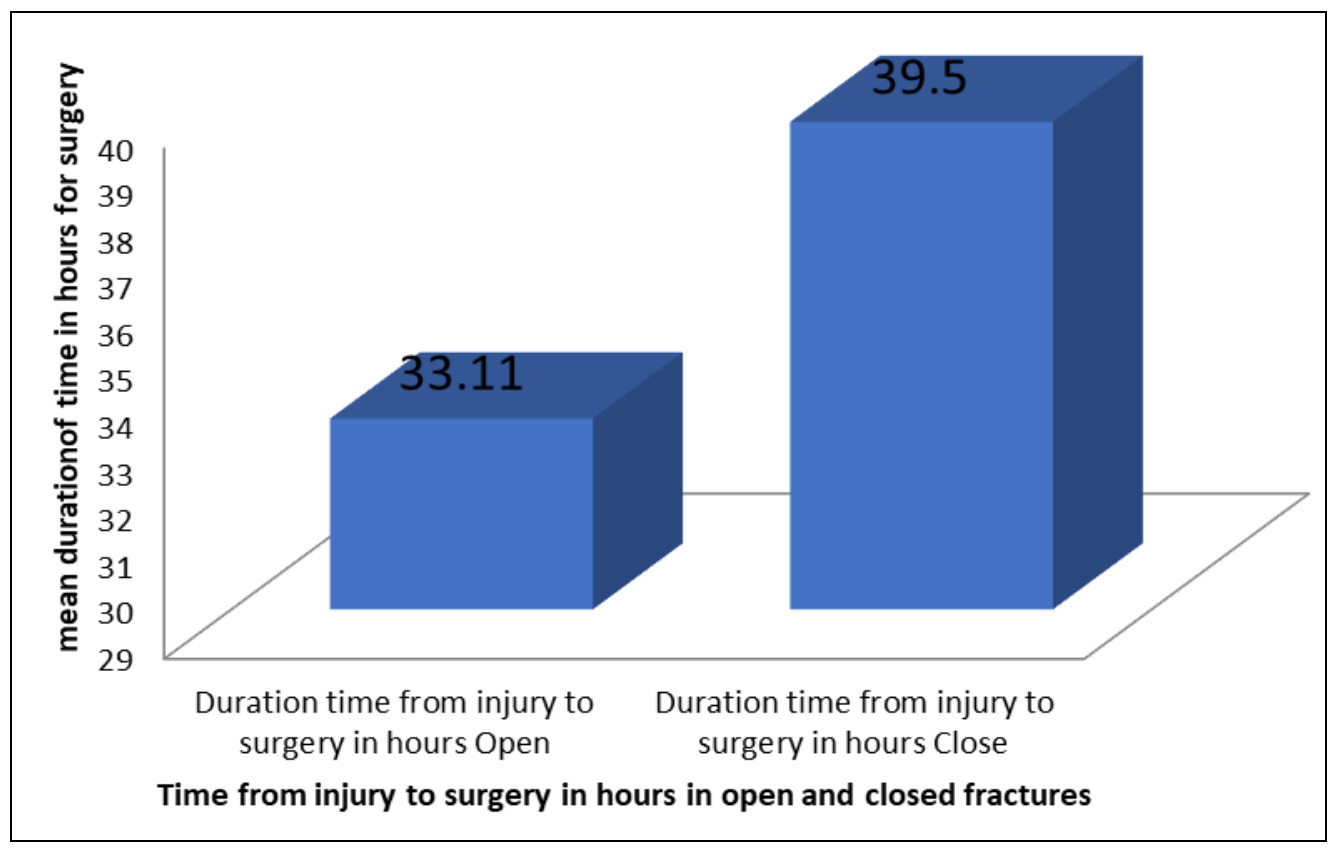

Fig 1: Mean duration of time in hours from injury to surgery in both Open and Closed fractures $\sim 769 \sim$ 


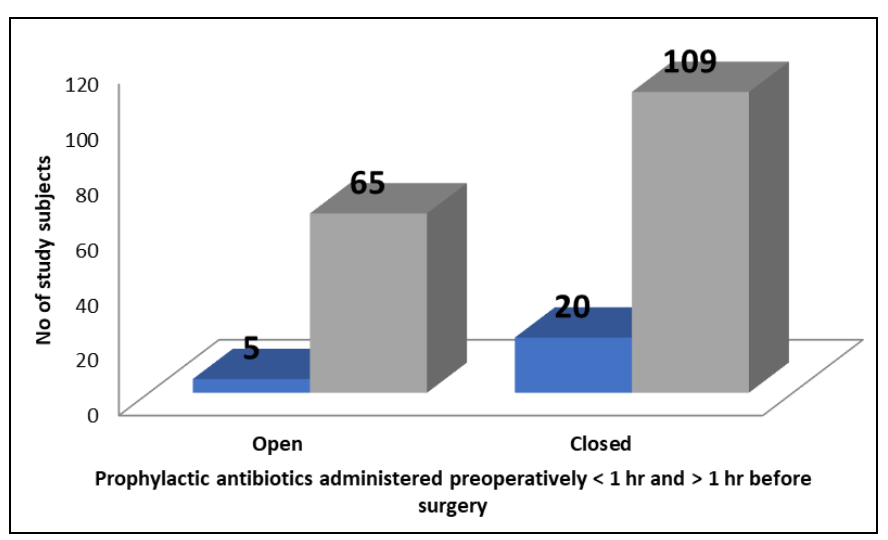

Fig 2: Depicting no of patients who received prophylactic antibiotics preoperatively with in one hour before surgery in Open and closed fracture categories

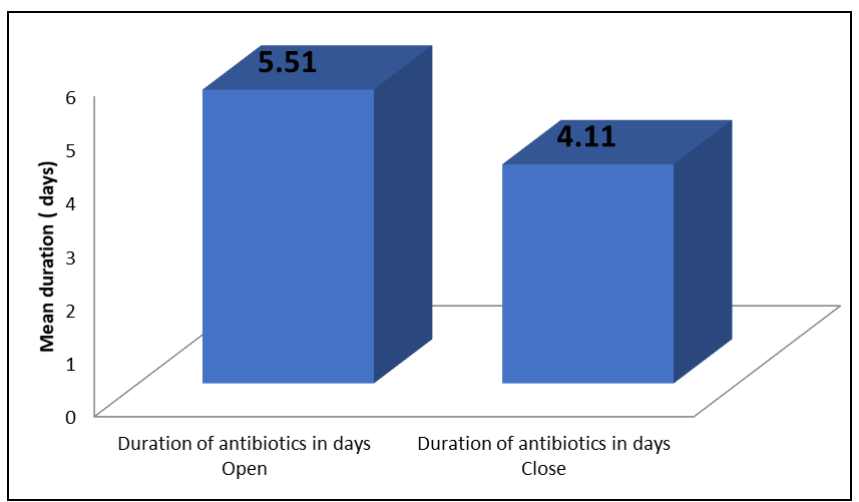

Fig 3: Depicting Average no of days that the antibiotics administered in post operative period

Rate of infection: The overall rate of infection within 12 months of surgery in our study was $11 \%$ (22participants). The incidence was higher in open fractures (17.1\%) (12 infections) compared to that of closed fractures (7.8\%) (10infections) $(\mathrm{p}<0.0001)$. Of the 12 infections in open fractures, 3 occurred in Gustillo type I fractures, 3 in type II, 1 in Type III A fractures and 5 infections in type IIIB open fractures. 3 of 16 patients (15.7\%) from Type 1 open fracture got infected, like wise 3 of 32 (9.4\%) patients from type II, 1 of $10(10 \%)$ from type IIIA and 5 of $9(55.6 \%)$ with type IIIB open fractures got infected with $\mathrm{P}$ value 0.01 suggesting as the severity of Gustilo type increases the infection rate following surgery increases (Fig 4).Of the 10 infections in closed fractures, 1 occurred in a Tscherne's Type 0 fractures, 2 in type 1 fractures, 6 in Type 2 and 1 in type 3 fractures (Fig 5), 1 of 9 patients( $11.1 \%$ ) from Type 0 Closed fractures got infected, like wise 2 of 73 (2.7\%) patients from type 1,6 of 42 (14.3\%) from type 2 and 1 of $5(20 \%)$ with type 3 Closed fractures got infected with $\mathrm{P}$ value 0.1 suggesting no significant association between the occurrence of infection and the severity of closed fracture classified as per Tscherne's classification. There were 15 deep infections noticed in our study (9 in open and 6 in closed fracture categories) and 7 superficial infections ( 3 in open and and 4 in closed fractures) with $\mathrm{P}$ value 0.652 indicating no significant association between occurrence of deep and superficial infections in either of the fracture types (table 4)

There were 8 early infections ( 4 in open and 4 in closed fractures), 5 delayed infections ( 2 in open and 3 in closed), and 9 late infections ( 6 in open and 4 in closed) noticed in our study with $(\mathrm{p}=0.598)$ suggesting no statistical significance between fracture type and occurrence of infection stratified by time (Table 5, Fig 8). It was observed that 7 patients $(31 \%)$ of 22 infected cases were smokers with 4 patients in closed fracture group and 3 in open fracture group. 15 of 22 infected cases (69\%) are non smokers with 10 patients in closed group and 5 patients in open group, with p value 0.060 suggesting no significant association between smoking and occurrence of infection in our study (Table 3, fig 6).

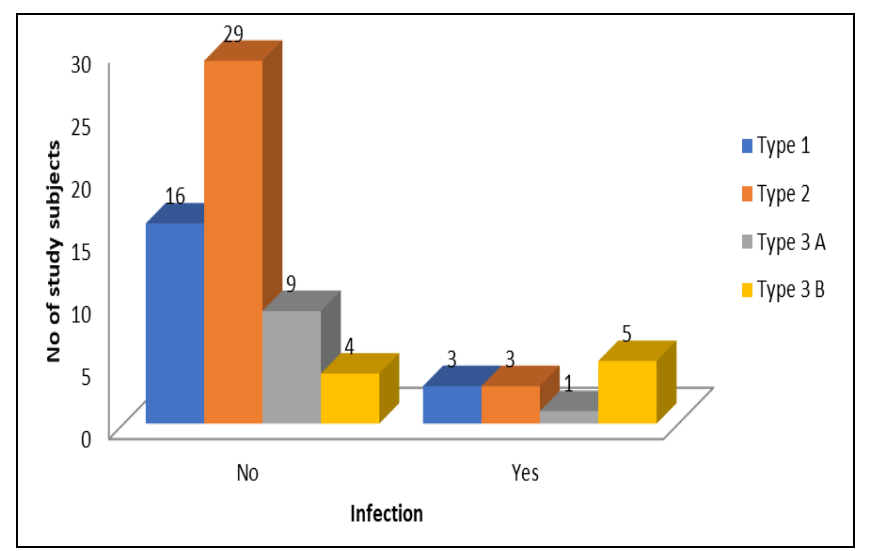

Fig 4: representing the number of infections in each Type of open fracture on right side of figure as opposed to total number of cases in each Type as per Gustilo classification on right.

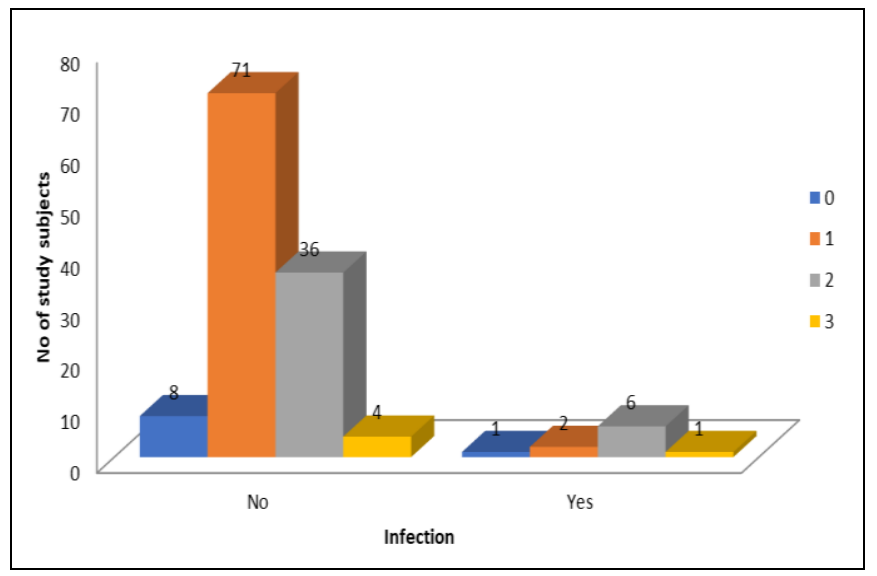

Fig 5: representing the number of infections in each Type of Closed fracture on right side of figure as opposed to total number of cases in each Type as per Tscherne's classification on left.

Table 3: depicting association between smoking and occurrence of infection in patients with both open and closed fractures

\begin{tabular}{|c|c|c|c|c|c|}
\hline Smoking History & No of Closed \# With Infection & No of Open \# With Infection & Total & Percentage & P value \\
\hline Smokers & 4 & 3 & 7 & $31 \%$ & \\
\hline Non Smokers & 10 & 5 & 15 & $69 \%$ & 0.060058655 \\
\hline Total & 14 & 8 & 22 & $100 \%$ & \\
\hline
\end{tabular}




\section{Chart Title}

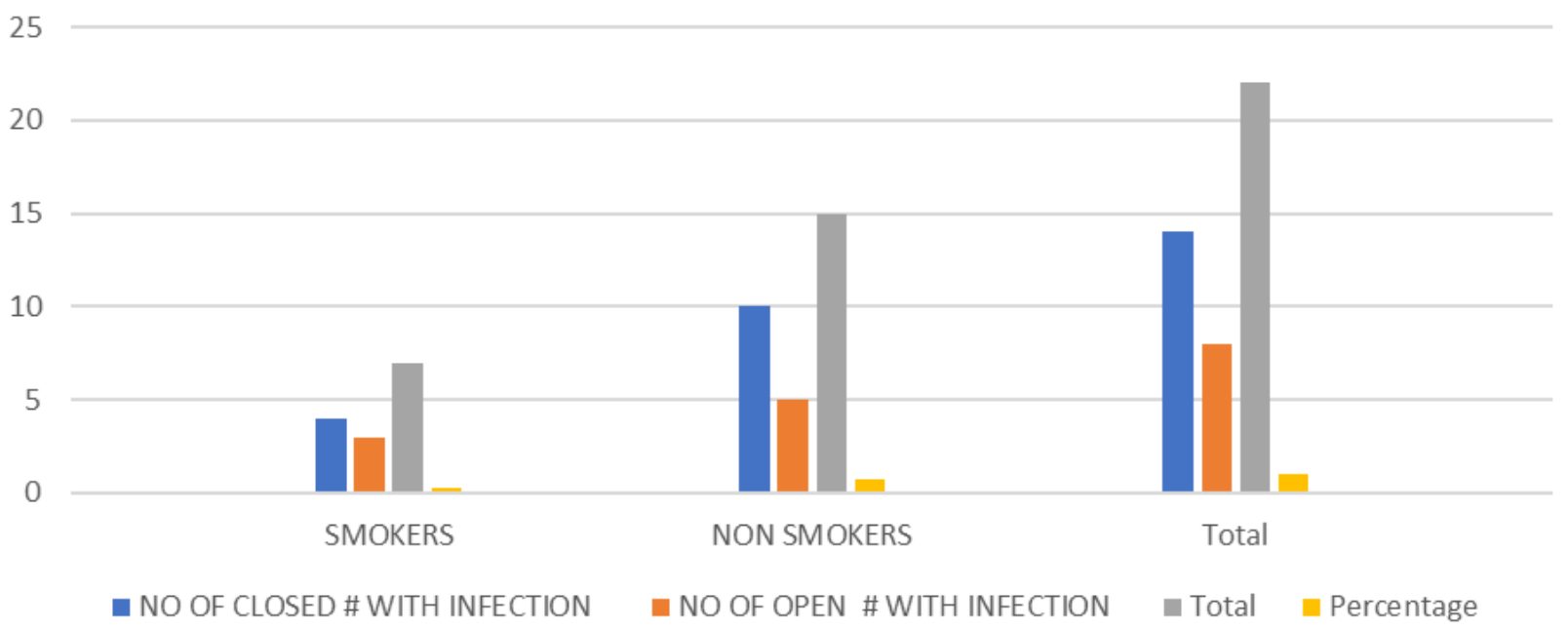

Fig 6: Depicting association between smoking and occurrence of infection in patients with both open and closed fractures.

Table 4: Depicting No of superficial infections, and deep infections, noticed in both open and closed fractures in our study.

\begin{tabular}{|c|c|c|c|c|c|}
\hline Type of infection & Open & Closed & Total & Chi square & p value \\
\hline \multirow{2}{*}{ Deep } & 9 & 6 & 15 & & \\
\cline { 2 - 4 } & $75.0 \%$ & $60.0 \%$ & $68.2 \%$ & & \\
\hline \multirow{2}{*}{ Superficial } & 3 & 4 & 7 & \multirow{3}{*}{0.566} & \multirow{2}{*}{0.652} \\
\cline { 2 - 4 } & $25.0 \%$ & $40.0 \%$ & $31.8 \%$ & & \\
\hline \multirow{2}{*}{ Total } & 12 & 10 & 22 & & \\
\cline { 2 - 4 } & $100.0 \%$ & $100.0 \%$ & $100.0 \%$ & & \\
\hline
\end{tabular}

*Superficial SSI - Infection involves only skin and subcutaneous tissue of incision

*Deep SSI - Infection involves deep tissues.

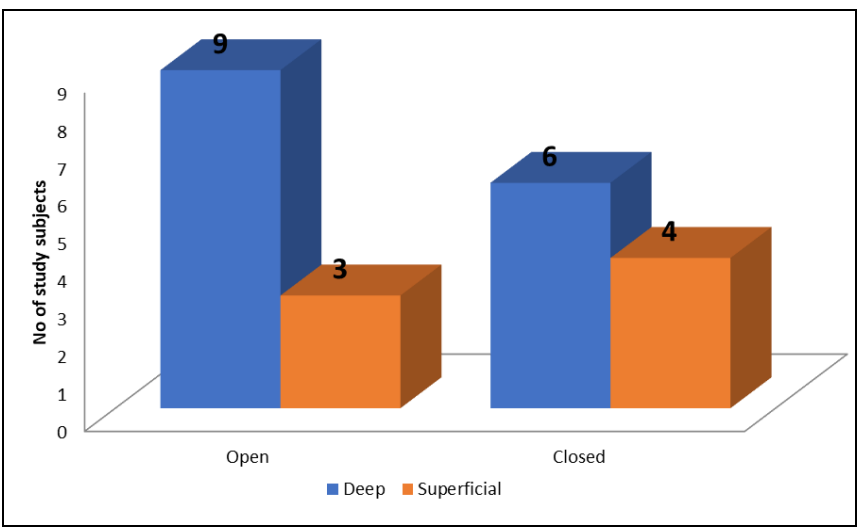

Fig 7: depicting No of superficial infections, and deep infections, noticed in both open and closed fractures in our study.

Table 5: showing number of early, delayed, and late infections noticed in our study population in both open and closed fracture types.

\begin{tabular}{|c|c|c|c|c|c|}
\hline $\begin{array}{l}\text { Type of } \\
\text { infection }\end{array}$ & Open & Closed & Total & $\begin{array}{c}\text { Chi } \\
\text { square }\end{array}$ & $\begin{array}{c}\mathbf{p} \\
\text { value }\end{array}$ \\
\hline \multirow{2}{*}{$\begin{array}{c}\text { Early }(<2 \\
\text { weeks })\end{array}$} & 4 & 4 & 8 & \multirow{8}{*}{1.027} & \multirow{8}{*}{0.598} \\
\hline & $33.3 \%$ & $40.0 \%$ & $36.4 \%$ & & \\
\hline \multirow{2}{*}{$\begin{array}{l}\text { Delayed ( } 2 \text { to } \\
10 \text { weeks) }\end{array}$} & 2 & 3 & 5 & & \\
\hline & $16.7 \%$ & $30.0 \%$ & $22.7 \%$ & & \\
\hline \multirow{2}{*}{$\begin{array}{c}\text { Late }(>10 \\
\text { weeks) }\end{array}$} & 6 & 3 & 9 & & \\
\hline & $50.0 \%$ & $30.0 \%$ & $40.9 \%$ & & \\
\hline \multirow{2}{*}{ Total } & 12 & 10 & 22 & & \\
\hline & $100.0 \%$ & $100.0 \%$ & $100.0 \%$ & & \\
\hline
\end{tabular}

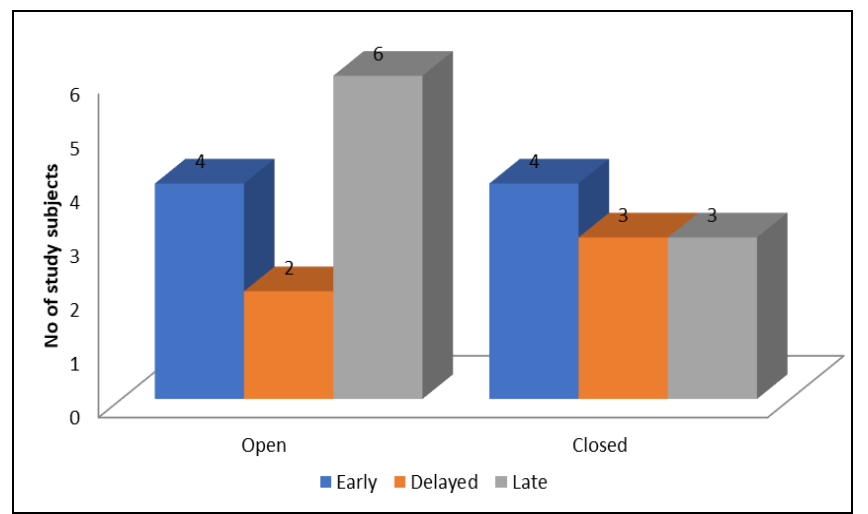

Fig 8: Showing number of early, delayed, and late infections noticed in our study population in both open and closed fracture types.

Infection characteristics and management: Symptoms of infection included discharge from surgical site, non healing surgical wound, erythema, and local pain noticed in $11.1 \%$ (22 patients). Discharge was predominantly sero purulent in $63.3 \%$ of individuals purulent in $27.3 \%$ and serous in $9.1 \%$ of infected individuals. Bacterial cultures were taken in all the participants with infections and positive bacterial cultures were found in $77.3 \%$ of the culture reports. The most commonly isolated organism in our study was Staphylococcus aureus $(59.1 \%)$ followed by Pseudomonas aeruginosa in $13.6 \%$ of cases. In about $22.7 \%$ culture reports were negative. Staph aureus was isolated in 7 out of 12 infected cases in open fractures and in 5 out of 10 cases in closed fracture category (Table 6 and Fig 9).

The mean ESR raise noticed in infected cases of open and closed fractures were $30.75 \pm 16.05 \mathrm{~mm} / \mathrm{hr}$ and $38.80 \pm 25.66$ respectively $(\mathrm{p}=0.38)$ which is not statistically significant. The average Total Leucocyte Count (TLC) found in our study in the infected cases among both open and closed fractures were 14,437 and 12,881 respectively. Majority of the infections were treated with IV antibiotics only $(50 \%)$ followed by implant removal and antibiotics in $36.4 \%$ and implant removal and fixation with external fixator device in $13.6 \%$ cases. (Table 7, Fig 10) 
Table 6: Shows the microorganisms isolated from the discharge of infected cases.

\begin{tabular}{|c|c|c|c|c|c|}
\hline Organism isolated & Open & Closed & Total & Chi square & p value \\
\hline \multirow{2}{*}{ Klebsiella pneumoniae } & 1 & 0 & 1 & \multirow{10}{*}{1.440} & \multirow{10}{*}{0.696} \\
\hline & $8.3 \%$ & $0.0 \%$ & $4.5 \%$ & & \\
\hline \multirow{2}{*}{ None } & 2 & 3 & 5 & & \\
\hline & $16.7 \%$ & $30.0 \%$ & $22.7 \%$ & & \\
\hline \multirow{2}{*}{ Pseudomonas aeruginosa } & 2 & 1 & 3 & & \\
\hline & $16.7 \%$ & $10.0 \%$ & $13.6 \%$ & & \\
\hline \multirow{2}{*}{ Staph aureus } & 7 & 6 & 13 & & \\
\hline & $58.3 \%$ & $60.0 \%$ & $59.1 \%$ & & \\
\hline \multirow{2}{*}{ Total } & 12 & 10 & 22 & & \\
\hline & $100.0 \%$ & $100.0 \%$ & $100.0 \%$ & & \\
\hline
\end{tabular}

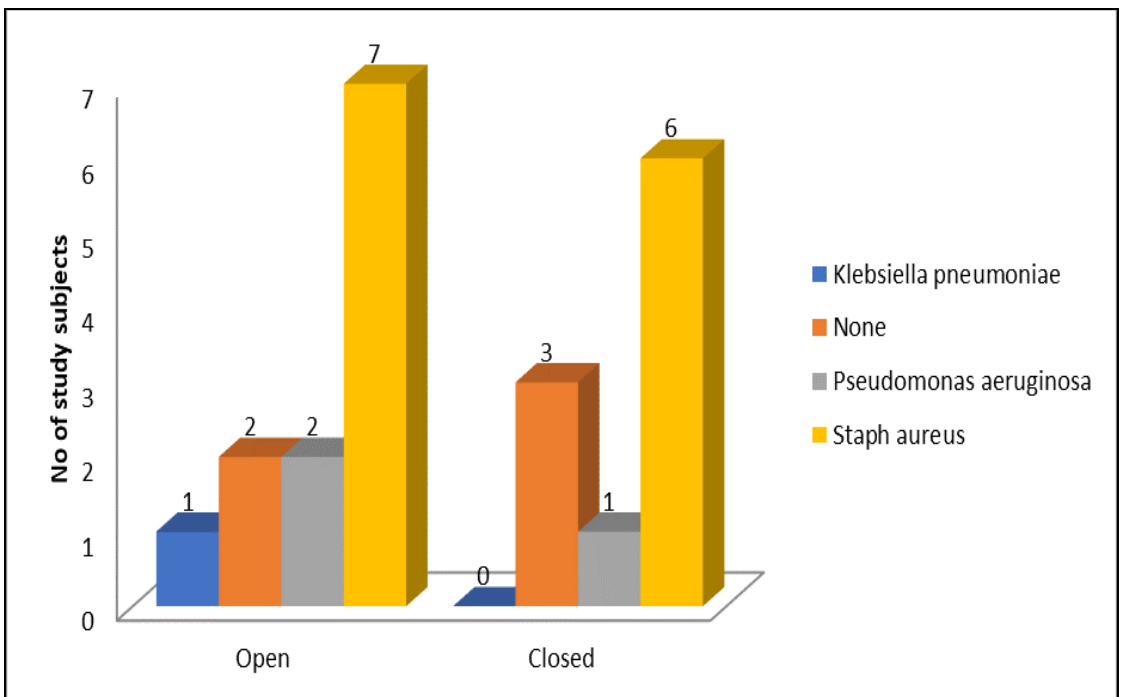

Fig 9: Shows the microorganisms isolated from the discharge of infected cases in open and closed fractures.

Table 7: Showing interventions done to manage the infected cases in our study

\begin{tabular}{|c|c|c|c|c|c|}
\hline Treatment given & Open & Closed & Total & Chi square & p value \\
\hline Imnlant remoual and fixation with External fixator & 1 & 2 & 3 & \multirow{8}{*}{0.749} & \multirow{8}{*}{0.688} \\
\hline mimprant removal and inxation witn external hxator & $8.3 \%$ & $20.0 \%$ & $13.6 \%$ & & \\
\hline \multirow{2}{*}{ Implant removal and IV antibiotics } & 5 & 3 & 8 & & \\
\hline & $41.7 \%$ & $30.0 \%$ & $36.4 \%$ & & \\
\hline \multirow{2}{*}{ IV antibiotics alone } & 6 & 5 & 11 & & \\
\hline & $50.0 \%$ & $50.0 \%$ & $50.0 \%$ & & \\
\hline \multirow{2}{*}{ Total } & 12 & 10 & 22 & & \\
\hline & $100.0 \%$ & $100.0 \%$ & $100.0 \%$ & & \\
\hline
\end{tabular}

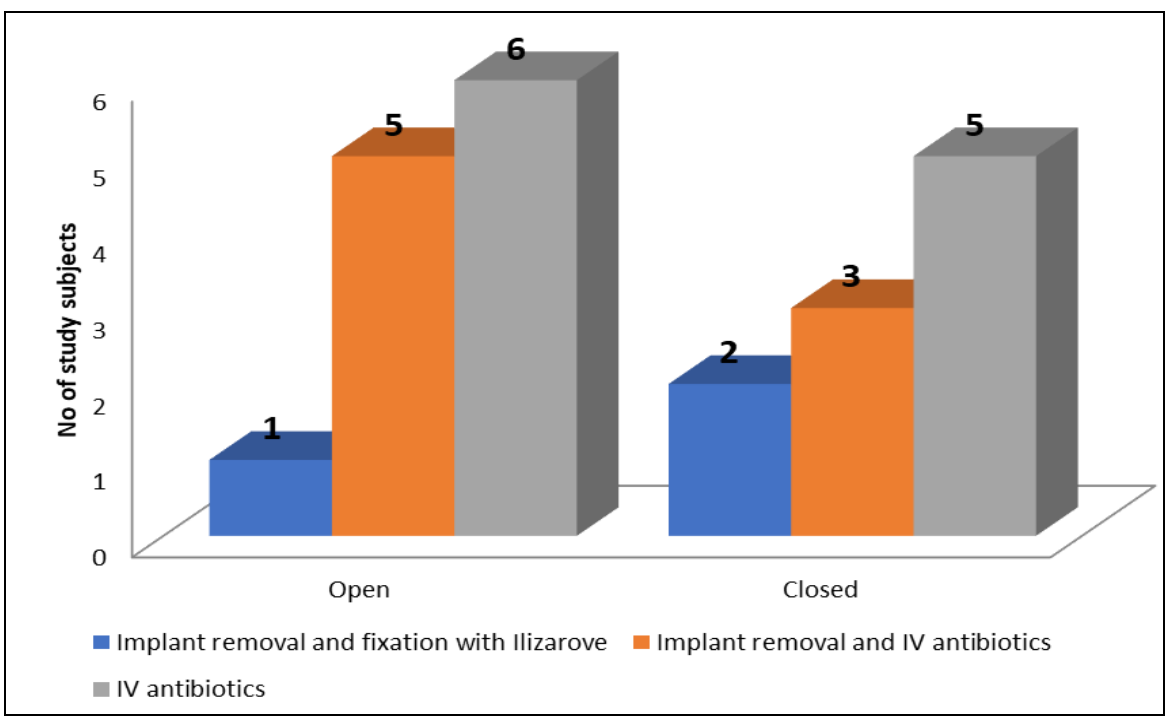

Fig 10: Showing interventions done to manage the infected cases in our study 


\section{Clinical pictures}
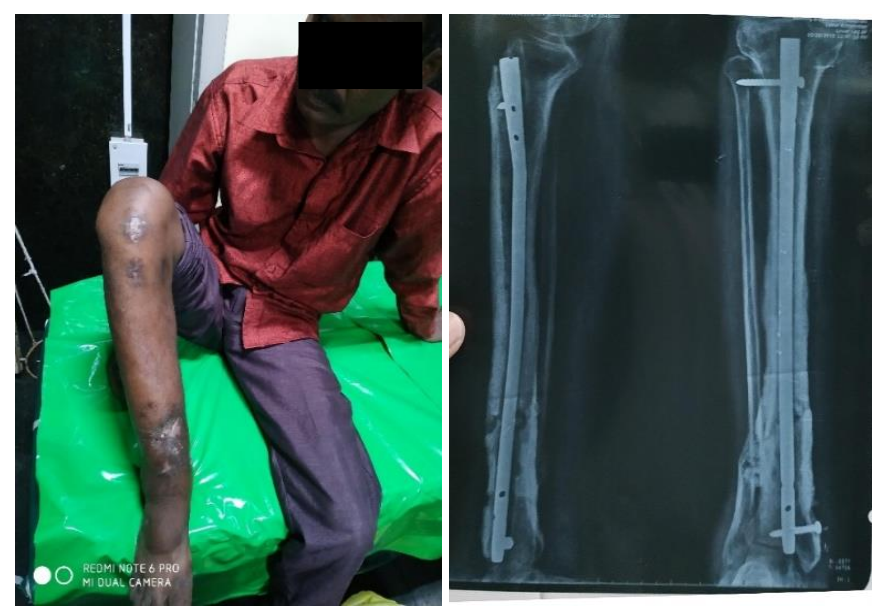

Photograph:1Infected limb with discharging sinus radiograph with infected nail
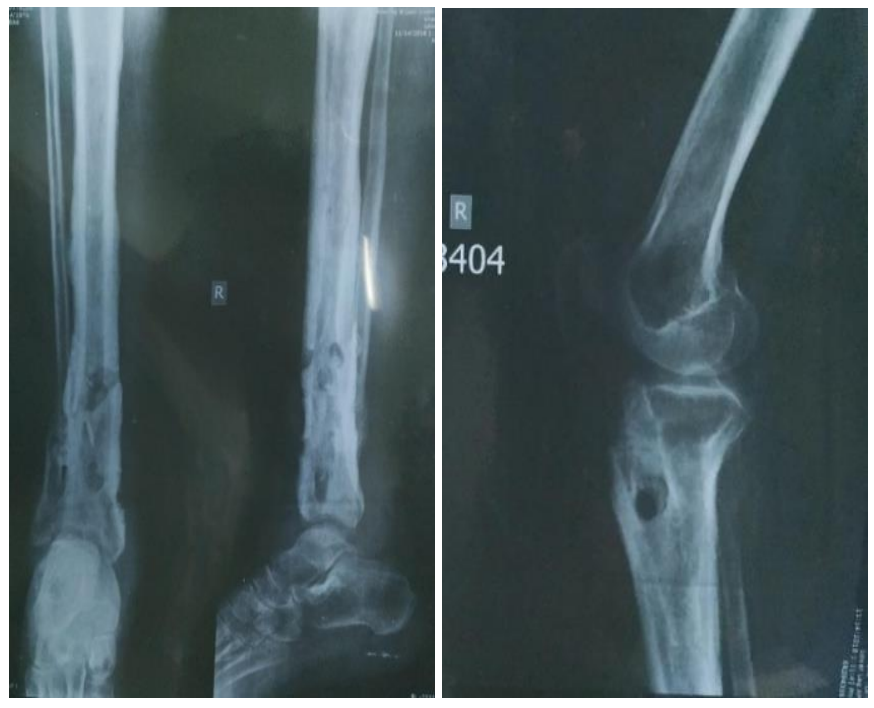

Radiograph taken Immediately After implant removal
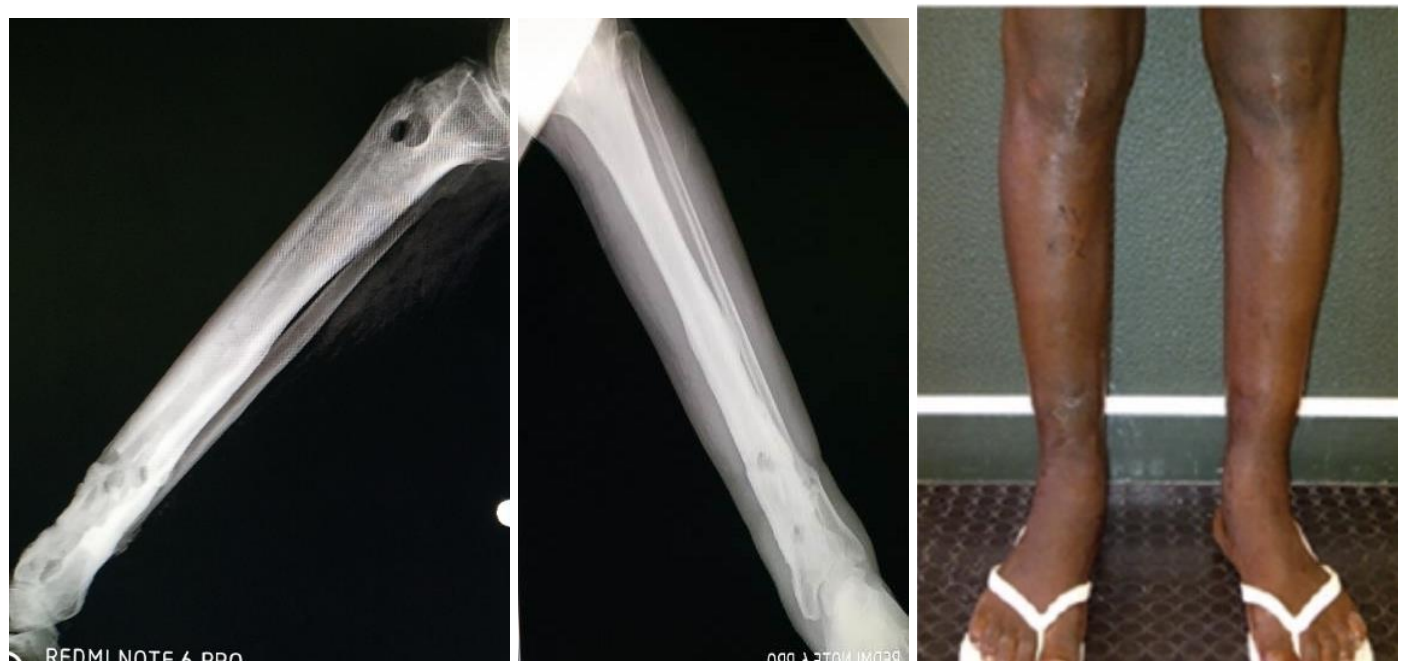

Post removal of implant and IV antibiotics radiograph and clinical picture with union at fracture Site and healed infected site.

\section{Discussion}

Following surgical management of tibial shaft fractures with ILN in a cohort of 199 participants operated at Victoria and Bowring and Lady Curzon Hospitals the rate of infection seen in closed and open fractures were $7.8 \%$ and $17.1 \%$, respectively. Infection was much higher to those seen in the large investigations of tibial shaft fractures in developed countries, which were $1.9 \%$ in closed fractures and $8.8 \%$ in open fractures ${ }^{[6]}$. Court brown et al in their study conducted in Edinburg observed that out of 459 patients treated for tibial fractures with Intra medullary nail,incidence of infection was $1.8 \%$ in closed fractures and about $9.5 \%$ in open fractures ${ }^{[7]}$. When compared with the infection rates in Low and middle income countries, infection rates seen in our study still stand high. As per study conducted by sven young et al, it was observed that overall infection rate following Intra medullary nailing of tibial fractures fall between 1.5 to $6.9 \%{ }^{[8]}$. A study conducted by Doshi et al., in various parts of India observed that overall infection rate was $2.9 \%$ (1.6\% in closed and $8 \%$ in open fractures of tibia treated with internal fixation). The high infection rates in our study is surprising, and it alerts us to raise a concern over various factors that need to be scrutinised that have a role in maintaining infection free environment in peri operative care of patient. The reasons for such high infection rate encountered in our research could be majority of participants sustaining injuries from motor vehicle accidents (95\%), with significant number of open fractures with possible contamination. Majority of patients experienced long duration of time from time since injury to surgery ( $33 \mathrm{hrs}$ for open and $40 \mathrm{hrs}$ for closed fractures). The typical time duration between injury and surgery in developed countries for open fractures has been reported to be $9.8 \mathrm{hrs}$, which is very much shorter than the time observed within our study ${ }^{[9]}$. Contributing factors may include patients living in rural areas of our state and unable to travel to a hospital in an appropriate time due to travel difficulties. It was observed that $31 \%$ infected cases were smokers and $69 \%$ were non smokers in both open closed fracture groups $(\mathrm{p}=0.060)$ smoking doesn't seem to have a significant role in our study suggesting no significant association between smoking and occurrence of infection in occurrence of infection as per our study. Despite the administration of prophylactic antibiotics for longer periods ie average of 5.5 days in open fractures and 4.41 days in closed fractures, infection rates remained high, hinting us about prolonged and irrational usage of antibiotics in our routine practice needs to be changed. AAOS recommends to stop prophylactic antibiotic post operatively after $24 \mathrm{hrs}$. Another important factor that should be kept in mind is the administration of right antibiotic in right doses preoperatively within 30 to 60 minutes to incision to maintain optimum plasma concentrations of antibiotic has been proposed to decrease the risk of infection by $29 \%{ }^{10}$.In our study $13 \%$ of 
the patient's were not given prophylactic antibiotic with in an hour of surgery due to unknown reasons, of which 5 patients $(22.7 \%)$ found to have infection, this may also could have been a contributing factor. As the Gustilo type increased the occurrence of infection rate found to be increased with highest rate seen in type III B fractures (41.7\% of cases). It's a reminder for us to be cautious in selecting internal fixation with ILN in such cases. Closed fracture type as per Tscherne's classification doesn't seem to increase the risk of infection as per our study. In our study the most common organism isolated from infective cases was Staph aureus (59.1\% of samples) a gram positive cocci and the next common was Pseudomonas aeruginosa (13.6\% cases) a gram negative bacilli, these findings are in agreement with the extensive study of Arciola et al (2005) ${ }^{[11]}$ and Khosravi et al (2009) ${ }^{[12]}$ as they noticed most common organism isolated from infected orthopaedic implants were Staphyloccus aureus and other significant pathogen being Pseudomonas aueriginosa. Berquist et al and Dan et al., found that $29.8 \%$ of hospitalized patients and $26.6 \%$ of hospital staff respectively act as carriers for Staph aureus ${ }^{[13,14]}$. Hence its important to select the antibiotic that is effective for prophylaxis specific to the hospital setup to reduce occurrence of infection. We observed no significant difference among occurrence of early, delayed, and late infections in open and closed fractures. The mean ESR levels observed in our study were 31 and 39 $\mathrm{mm} / \mathrm{hr}$ in open and closed fractures categories which is of diagnostic significance as it is a very useful screening investigation. The elevation of ESR has high sensitivity and may have a low specificity. Values of more than 30 or $35 \mathrm{~mm}$ per hour are considered to be abnormal and indicative of infection unless proven otherwise ${ }^{[72]}$. Surgical site Current guidelines outline drainage, debridement, and specific antibiotic prescription as the hallmark treatment regimen for SSI; however infection management in our study primarily consists

of antibiotics alone in $50 \%$ of cases, implant removal and antibiotics in $36.4 \%$ of cases and implant removal and fixation with external fixator in $13.6 \%$ cases. This study is strengthened by by its prospective design and we have clearly defined eligibility criteria prior to study initiation to ensure that all included participants were an appropriate representation of the target population. Additional strength was gained through the decent sample size. We were also able to capture details regarding current standardized treatment methods and antibiotic regimens that were followed in department of orthopaedics at our institution. The significant number of infections in our study ie $22(11 \%)$ increases the power of our statistical significance, as the sample size of infections is reasonably high compared to other similar studies. As the inclusion of only tibial shaft fractures, treated with ILN the results may not be accurate for drawing conclusions for other methods of fixation and for other bones in the body. The potential limitation of this study is that the hospitals where this study conducted may not be representative all Indian hospitals, as there are large and modern facilities with experience in clinical research and also other setups with poor facilities. There exist several risk factors for surgical site infections that were not investigated in our study. Future studies should aim to explore other risk factors and determine if there is a relationship between organism isolated from wound of open fractures before debridement versus organism isolated from the infected cases following surgery in open cases, and also to study various pre, intra and post operative factors that influence the infection rates, there is also a need for studying bacteriological profiles in the instance of surgical site infections, as there is always a possibility of infection with more than one organism and their antibiotic resistance patterns in each setup. This is one of the first studies to our knowledge to provide a thorough overview of the incidence of infection, prophylactic antibiotic use, infection management options, for the patients who undergo internal fixation of a tibia fracture with ILN in India exclusively. This study allows for the calculation of required subjects for additional studies in this area of research.

\section{Conclusion}

Despite all the necessary measures taken at each step to our knowledge, infection rates in our study found to be quite high. It comes as a reminder to rethink where could be the possible loopholes leading to such higher rates of infection. As the infection control and prevention requires us to take strict actions at multiple levels the responsibility lies not only with people directly associated with the patient's treatment but also with entire health care personnel working in our setup at their own capacities. Providing better travel facilities would reduce time gap between injury to hospital admission and thereby surgery. Addressing the patient related factors such as improving general condition of the

patient by treating other associated systemic diseases, improving the nutritional status, educating on abstinence from smoking and alcoholism etc should be considered for a better outcome. Operating surgeon must ensure peri operative precautions be taken strictly in terms meticulous administration of prophylactic antibiotics, by observing strict sterile techniques to be followed by the team members in handling drapes, instrumentation, implants etc., Work environment must be monitored for better staff compliance, air handling system, restriction of unnecessary trafficking in the operating rooms. Despite administering prophylactic antibiotics for much longer period than the recommended doses, infection rate is still high. Future research should aim to identify the best practices at various levels to decrease the infection rates, for management of infection and for prophylactic antibiotic use. There should be a strict treatment algorithm drafted to be followed by the entire hospital.

\section{References}

1. Joshipura MK, Shah HS, Patel PR, Divatia PA, Desai PM. Trauma care systems in India. Injury 2003;34(9):686-92.

2. Court-Brown CM, Caesar B. Epidemiology of adult fractures: a review. Injury 2006;37:691-7.

3. Rudolf I Matthew, Fractures of lower extremity, S. Terren Canale, James H Beaty: Campbell's Operative Orthopaedics 2013;3:2650.

4. Saris CG, Bastianen CA, Mvan Swieten EC, Wegdam $\mathrm{HH}$. Infection rate in closed fractures after internal fixations in a municipal hospital in Ghana. Trop Doct 2006;36(4):233-5.

5. CDC. Surgical Site Infection (SSI) Event. Procedureassociated Module 2015;9-1-9-26.

6. Bhandari M, Guyatt G, Tornetta 3rd P, Schemitsch EH, Swiontkowski M, Sanders D et al. Randomized trial of reamed and unreamed intramedullary nailing of tibial shaft fractures. J Bone Joint Surg Am 2008;90(12):256778.

7. Court-Brown CM, Keating JF, McQueen MM. Infection after intramedullary nailing of the tibia incidence and protocol for management..,J Bone Joint Surg [Br] 
1992;74-B:770-4.

8. Young S, Lie SA, Hallan G et al. World J Surg 2013;37:3-6

9. The Flow Investigators. A Trial of Wound Irrigation in the Initial Management of Open Fracture Wounds. N Engl J Med. 2015;373:2629-41.

10. Young S, Lie SA, Hallan G, Zirkle LG, Engesaeter LB, Havelin LI. Risk factors for infection after 46,113 intramedullary nail operations in low-and middleincome countries. World J Surg 2013;37(2):349-55.

11. Arciola CR, An YH, Campoccia D, Donati ME, Montanaro L. Etiology of implant orthopedic infections: A survey on 1027 clinical isolates. Int. J. Artif. Organs 2005;28:1091-10100.

12. Khosravi AD, Ahmadi F, Salmanzadeh S, Dashtbozorg A, Abasi Montazeri E. Study of Bacteria Isolated from Orthopedic Implant Infections and their Antimicrobial Susceptibility Pattern. Research Journal of Microbiology 2009;4:158-163.

13. Bergqvist S. Observations concerning the presence of pyogenic staphylococci in the nose and their relationship to the antistapholysin titre. Acta Med Scand 1950;136:343-50.

14. Dan M, Moses Y, Poch F, Asherov J, Gutman R. Carriage of methicillin-resistant S. aureus by nonhospitalized subjects in Israel. Infection 1992;20:332-5. 7 